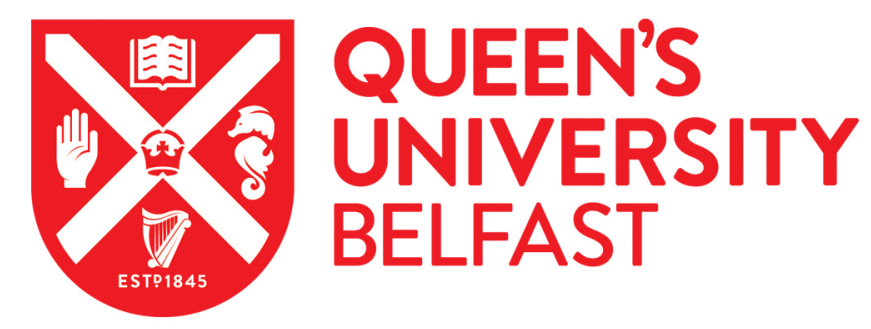

\title{
Observation of the de Vries behavior in SmA* phase of a liquid crystal using polarised Raman scattering and infrared spectroscopy
}

Kocot, A., Vij, J. K., Perova, T. S., Merkel, K., Swaminathan, V., Sreenilayam, S. P., Yadav, N., Panov, V. P., Stevenson, P. J., Panov, A., \& Rodriguez-Lojo, D. (2017). Observation of the de Vries behavior in SmA* phase of a liquid crystal using polarised Raman scattering and infrared spectroscopy. Journal of Chemical Physics, 147(9), [094903]. https://doi.org/10.1063/1.4999792

Published in:

Journal of Chemical Physics

Document Version:

Publisher's PDF, also known as Version of record

Queen's University Belfast - Research Portal:

Link to publication record in Queen's University Belfast Research Portal

Publisher rights

Copyright 2017 AIP. This work is made available online in accordance with the publisher's policies. Please refer to any applicable terms of use of the publisher.

\section{General rights}

Copyright for the publications made accessible via the Queen's University Belfast Research Portal is retained by the author(s) and / or other copyright owners and it is a condition of accessing these publications that users recognise and abide by the legal requirements associated with these rights.

Take down policy

The Research Portal is Queen's institutional repository that provides access to Queen's research output. Every effort has been made to ensure that content in the Research Portal does not infringe any person's rights, or applicable UK laws. If you discover content in the Research Portal that you believe breaches copyright or violates any law, please contact openaccess@qub.ac.uk. 


\section{Observation of the de Vries behavior in SmA" phase of a liquid crystal using polarised Raman scattering and infrared spectroscopy}

A. Kocot, J. K. Vij, T. S. Perova, K. Merkel, V. Swaminathan, S. P. Sreenilayam, N. Yadav, V. P. Panov, P. J. Stevenson, A. Panov, and D. Rodriguez-Lojo

Citation: The Journal of Chemical Physics 147, 094903 (2017); doi: 10.1063/1.4999792

View online: https://doi.org/10.1063/1.4999792

View Table of Contents: http://aip.scitation.org/toc/jcp/147/9

Published by the American Institute of Physics

\section{Articles you may be interested in}

Coarsening and aging of lattice polymers: Influence of bond fluctuations

The Journal of Chemical Physics 147, 094902 (2017); 10.1063/1.4991667

Transferable coarse-grained model for perfluorosulfonic acid polymer membranes

The Journal of Chemical Physics 147, 094904 (2017); 10.1063/1.4986287

Dynamic Kerr effect in a strong uniform AC electric field for interacting polar and polarizable molecules in the mean field approximation

The Journal of Chemical Physics 147, 094501 (2017); 10.1063/1.4995021

Knotting probability of self-avoiding polygons under a topological constraint

The Journal of Chemical Physics 147, 094901 (2017); 10.1063/1.4996645

Length and sequence relaxation of copolymers under recombination reactions

The Journal of Chemical Physics 147, 094905 (2017); 10.1063/1.5001021

Thermodynamic responses of electronic systems

The Journal of Chemical Physics 147, 094105 (2017); 10.1063/1.4999761

\section{AIP| The Jounal of Chemical Physics}

PERSPECTIVES 


\title{
Observation of the de Vries behavior in SmA* phase of a liquid crystal using polarised Raman scattering and infrared spectroscopy
}

\author{
A. Kocot, ${ }^{1}$ J. K. Vij, ${ }^{2, a)}$ T. S. Perova, ${ }^{2,3}$ K. Merkel, ${ }^{4}$ V. Swaminathan, ${ }^{2}$ S. P. Sreenilayam, ${ }^{2}$ \\ N. Yadav, ${ }^{2}$ V. P. Panov, ${ }^{2}$ P. J. Stevenson, ${ }^{5}$ A. Panov, ${ }^{5}$ and D. Rodriguez-Lojo ${ }^{5}$ \\ ${ }^{1}$ Institute of Physics, Silesian University, Katowice, Poland \\ ${ }^{2}$ Department of Electronic and Electrical Engineering, Trinity College Dublin, The University of Dublin, \\ Dublin 2, Ireland \\ ${ }^{3}$ ITMO University, Kronverkskiy pr. 49, Saint Petersburg, Russia \\ ${ }^{4}$ Central Mining Institute, Katowice, Poland \\ ${ }^{5}$ School of Chemistry and Chemical Engineering, Queen's University Belfast, Northern Ireland, United Kingdom
}

(Received 6 April 2017; accepted 10 August 2017; published online 1 September 2017)

\begin{abstract}
Two approaches exist in the literature for describing the orientational distribution function (ODF) of the molecular directors in $\mathrm{SmA} *$ phase of liquid crystals, though several models are recently proposed in the literature for explaining the de Vries behaviour. These ODFs correspond to either the conventional unimodal arrangements of molecular directors arising from the mean field theory that leads to the broad or sugar-loaf like distribution or to the "diffuse-cone-shaped" type distribution proposed by de Vries. The hypothesis by de Vries provides for a realistic explanation as to how at a molecular level, a first-order $\mathrm{SmA}^{*}$ to $\mathrm{SmC}$ * transition can occur where the uniform molecular director azimuthal distributions condense to values lying within a narrow range of angles; finally these condense to a single value while at the same time ensuring a little or no concomitant shrinkage in the layer spacing. The azimuthal distribution of the in-layer directors is probed using IR and polarized Raman spectroscopic techniques. The latter allows us to obtain the ODF and the various order parameters for the uniaxial and the biaxial phases. Based on the results of these measurements, we conclude that the "cone-shaped" (or volcano-shaped) de Vries type of distribution can most preferably describe SmA* where "a first-order phase transition from SmA* to SmC*" and a low layer shrinkage can both be easily explained. Published by AIP Publishing. [http://dx.doi.org/10.1063/1.4999792]
\end{abstract}

\section{INTRODUCTION}

A rod-shaped molecular system may form orthogonal $\mathrm{SmA}$ and/or tilted SmC liquid crystalline (LC) phases. These comprise two-dimensional orientationally ordered fluid states with one-dimensional density wave. The phase structure thus is regarded as an arrangement of rod-shaped molecules in smectic layers where the molecules can fluctuate, though these layers are not as well defined as in layered solid crystals. As in nematic LCs, the director $\hat{\boldsymbol{n}}$ in smectics is defined as the averaged direction of the long molecular axes of the constituent molecules. The orientational order parameters can describe the orientational distribution function (ODF) of the long molecular axes around the director; the existence of the smectic layers is specified by the positional order parameter. In the SmA phase, the director $\hat{\boldsymbol{n}}$ is parallel to the smectic layer normal $\hat{\boldsymbol{e}}$, while $\hat{\boldsymbol{n}}$ is tilted at an angle $\theta$ to $\hat{\boldsymbol{e}}$ in the $\mathrm{SmC}$ phase. When the molecules are optically active, the chiral smectic $\mathrm{C}$ phase ( $\left.\mathrm{SmC}^{*}\right)$ appears and the spontaneous polarization $\boldsymbol{P}_{S}$ emerges, locally normal to the tilt plane. The tilt plane is formed by the vectors $\hat{\boldsymbol{e}}$ and $\hat{\boldsymbol{n}}$. Correspondingly smectic $\mathrm{A}$ and $\mathrm{C}$ phases are denoted as SmA* and $\mathrm{SmC}^{*}$, the star indicates that constituent molecules are chiral. The chirality leads to a lack of mirror symmetry in a plane at right angles to the $\mathrm{C}_{2}$ axis. In the bulk LC's, the director forms a helicoidal structure in which the helical axis is parallel to $\hat{\boldsymbol{e}}$,

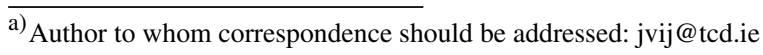

helical pitch lies usually in the $\mu \mathrm{m}$ range. The $\mathrm{SmA}^{*}-\mathrm{SmC}^{*}$ phase transition is driven mainly by the intermolecular interactions that produce $\mathrm{SmC}^{*}$ but not by the ferroelectric coupling between the permanent dipole moments. Thus the tilt angle, and not the spontaneous polarization in $\mathrm{SmC}^{*}$, is the primary order parameter.

More recently, the SmA phase in the bulk of some smectic LCs is reported to be of the de Vries type with a little layer-shrinkage at the SmA to SmC transition. De Vries in 1977 proposed that molecules in the SmA phase of some smectic LCs are tilted in a particular direction but the tilt direction (specified by the azimuthal angle) varies randomly from one layer to the next. ${ }^{1}$ De Vries model predicts $\mathrm{SmC}_{\mathrm{R}}$ * phase, where $\mathrm{R}$ stands for the randomized azimuthal angle that varies randomly from one layer to the next. Such a phase was first discovered by Panarin et al. ${ }^{2}$ This phase is also analogous to the sliding phase predicted by O'Hern, Lubensky, and Toner. ${ }^{3}$ Leadbetter and Norris ${ }^{4}$ made two important observations based on their $\mathrm{x}$-ray scattering results of a new class of nematic and smectic LCs synthesized by George Gray's group in Hull, United Kingdom. These are that (i) the orientational order parameter in the SmA phase in a class of compounds is much lower than in the same phase of most other compounds and (ii) the thickness of the smectic layer in the SmA phase is significantly lower than the extended length of the molecule. Based on the experimental results of the smectic layer thickness obtained using X-ray scattering by A. de Vries and based 
on an advanced pre-print of the Leadbetter and Norris article, de Vries gave a second model ${ }^{5}$ different from his previous one, in order to explain these unusual experimental results of the low layer shrinkage in the SmC phase. In his second model, it was suggested that the local in-layer directors in SmA are tilted in one direction due to the existence of a finite shortrange correlation length. However the tilting sense specified by the azimuthal angle varies within a single layer in this phase. The short-range correlations increase to long-range ones as the $\mathrm{SmA}$ to $\mathrm{SmC}$ transition temperature is approached. Both de Vries models in their ideal manifestations should lead to the "diffuse-cone shaped" ODF. However, it is not possible to distinguish macroscopically between the validity of one model against the second due to the inability of being able to discriminate the experimental results with predictions from these two models. Whether the correlated tilting directions are randomly oriented either (i) within a single layer or (ii) from layer to layer, and/or both, these cannot be easily discerned from the test of experimental results with the models. Nevertheless if the correlation length in the second model is not sufficiently large and the azimuthal distribution lacks complete randomness, then we could in principle obtain sugar-loaf or broad volcano type ODF in the SmA phase. These models were suggested at a time when mostly achiral molecules constituted smectic phases of LCs.

The SmA* phase of chiral de Vries smectics is found to exhibit unusual properties. These include the observations of (i) large electro-clinic effect, (ii) first-order $\mathrm{SmA}^{*}$ to $\mathrm{SmC}^{*}$ transition, and (iii) a low layer-shrinkage in the temperature range of the $\mathrm{SmC}^{*}$ phase relative to the layer thickness at the $\mathrm{SmA}^{*}-\mathrm{SmC} *$ phase transition temperature. Lagerwall and Giesselmann reviewed several models in the literature and in doing so, they introduced two ODF's: $f(\theta, \phi)$ and $f\left(\theta^{\prime}, \phi^{\prime}\right){ }^{6}$ Here $f(\theta, \phi)$ describes the distribution of the long molecular axes with respect to the director $\hat{\boldsymbol{n}}$, and $f\left(\theta^{\prime}, \phi^{\prime}\right)$ describes their distribution with respect to the smectic layer normal $\hat{\boldsymbol{e}}$. These two ODFs thus differ from each other in terms of a difference in the reference direction of the directors distribution. An explanation of the observed behavior in terms of the various models including the molecular mean-field model of Gorkunov et al. ${ }^{7}$ is given. The latter showed that a variation in the interaction potential could lead to both (i) the de Vries as well as to the conventional $\mathrm{SmA}^{*}$ phase, and also to (ii) the first order $\mathrm{SmA}^{*}-\mathrm{SmC} *$ phase transition being observed in some compounds. Saunders et al. explained the de Vries behaviour for both first and the second order $\mathrm{SmA}^{*}-\mathrm{SmC}^{*}$ phase transitions in smectic liquid crystals by parameterising the coefficients of the Landau expansion. ${ }^{8}$ Shen et al. ${ }^{9}$ introduced an interesting variation to the de Vries diffuse-cone model by extending the earlier model by Clark et al.,${ }^{10}$ whereby the cone-angle is restricted to lie in between the two limiting values of the tilt angle depending on the material but independent of temperature. ${ }^{9}$ The lower limit in the cone angle was found from the results of large increase in the birefringence with the field and the upper-limit depended on an increase in the saturated tilt angle by the electric field applied across a planar-aligned cell. The actual angle varied (largely though the electro-clinic effect) in between these two limiting values, depending on the temperature and the electric field applied. The de Vries characteristics such as (a) the field dependency of the birefringence and of (b) the tilt angle were first explained using the Fukuda-Langevin-Debye model ${ }^{11}$ with partial success. In the generalised version of their model, Shen et al. expressed the free-energy in terms of both linear and quadratic terms in the electric field. ${ }^{9}$

Here we use the Raman scattering to investigate an already proven de Vries smectic liquid crystal to obtain the ODF of the molecular directors in the $\mathrm{SmA}^{*}$ phase and to find whether the ODF is sugar-loaf [Fig. 1(a)] or is diffuse-cone [Fig. 1(b)]. Raman scattering refers to inelastic scattering of light caused by the interaction of incident beam of light with the material medium. Raman spectroscopy is used in many varied fields including such applications where non-destructive, microscopic, and chemical tests in terms of their images can directly be obtained. In our experiment, the scattered light arises mainly from the vibrational modes of molecules. The photons of the laser light are incident on the material, the spectral lines emerge in scattered light, wavelengths of which are both longer (Stokes line, comparatively of large intensity) and shorter (anti-Stokes line, rather of weak intensity) than that of the incident light. In the Raman spectra, the molecular vibrational bands for the Stokes lines appear in the spectra. These spectral lines correspond to the changes in polarizability with respect to the normal position coordinate for a given vibrational band.

A method of obtaining $\left\langle P_{2}\right\rangle$ and $\left\langle P_{4}\right\rangle$ from the Raman spectra was first established by Jen et al. ${ }^{12}$ for LCs in the nematic and smectic A phase. A methodology was developed for obtaining the uniaxial order parameters in nematic and smectic phases of a LC in terms of the depolarization ratio. Here the measurements of scattered light for the two orthogonal polarizations of light (parallel and perpendicular to the polarization of the light) incident normally on the medium were considered. The Raman method was subsequently extended by Jones et al. ${ }^{13}$ to include an extra variable in the experiment: angle that the director or the reference direction in a cell makes with the polarization direction of the incident light. The technique for both forward and backward scattered intensities of light in altered forms was used for determining the uniaxial and biaxial order parameters for the nematic and smectic phases..$^{13,14}$ Even before the studies of Jones et al. were finalized, Hayashi et al. ${ }^{15,16}$ independently presented a more general vision of the polarized Raman scattering technique and established a method of finding the order parameters. They took into account the polarization angle dependency of the depolarization ratio. This was also included in the methodology by Jones et al. ${ }^{13}$ at a later stage.

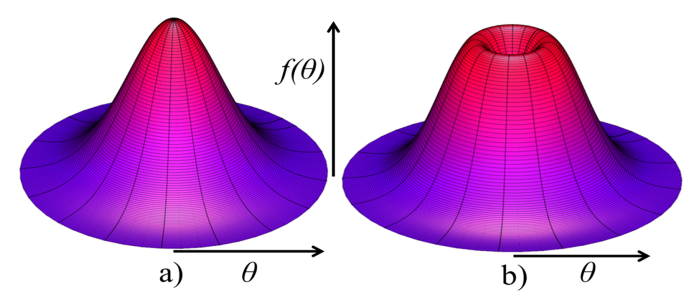

FIG. 1. Schematic representation of the ODF in the SmA phase. (a) Sugarloaf (b) diffuse cone or volcano shaped. These models are independent of whether the constituent molecules are chiral or achiral. 
However, Hayashi et al. had also included the corrections arising from birefringence and the refractive effects of the LC under investigation. ${ }^{15,16}$ Sanchez-Castillo et al. ${ }^{17}$ generalized the Raman method by including the biaxial order parameters and the correction terms introduced by Hayashi et al. They showed that their methodology reduces to that developed by Hayashi et al. ${ }^{15,16}$ when values of the biaxial order parameters are equated to zero as well as the tilt of the reference direction in the laboratory system relative to the layer normal is zero.

By using the Raman polarized spectroscopic technique, one can obtain, at least in principle, a set of parameters that govern the de Vries behaviour, thus allowing for a comparison of results with those deduced from the several models in the literature to take place. From such comparisons, it may be possible to select the most appropriate model for describing the ODF of the SmA* and SmC* phases of chiral smectics. As already stated above, the polarized Raman scattering methodology developed by Hayashi et al. ${ }^{15,16}$ can usefully give uniaxial, second $\left\langle P_{2}\right\rangle$ and the fourth $\left\langle P_{4}\right\rangle$ order orientational order parameters for the SmA* and SmC* phases of LCs. (for SmC*, the reference direction is shifted to lie along the optic axis/tilt direction). $\left\langle P_{2}\right\rangle$ and $\left\langle P_{4}\right\rangle$ for a prototypal de Vries TSiKN65 compound were found to be extremely low, ${ }^{18,19}$ not only in the absence of the external field but also when a large electric field was applied across a planar-aligned cell. By using their newly developed theory, Sanchez-Castillo et al. ${ }^{17}$ investigated a supposedly de Vries compound 9HL by polarised Raman and X-ray scattering techniques, results of which supported a "sugar loaf" ODF for its SmA* phase. Surprisingly, no qualitative difference in the ODF for the $\mathrm{SmA}^{*}$ phase was found for supposedly ideal de Vries smectic against the conventional smectic. A possible conclusion can be that 9HL, in spite of it having a small layer shrinkage and a large electro-clinic effect, is not a good candidate for "de Vries smectic." The ODFs for both the conventional and the twist-bend nematic phase of LC dimers were reported recently using Raman spectroscopy; ${ }^{20}$ results of the heliconical angle at a particular temperature agreed with those given by the birefringence measurements.

In this paper, Raman scattering and IR spectroscopy are used to determine the azimuthal distribution of the in-layer directors in a heptamethyltrisiloxane derivative compound, $\mathrm{MSi}_{3} \mathrm{MR} 11$, the chemical structure of which is given in Sec. II. This compound was previously found to exhibit a large electroclinic effect and confirmed to have the de Vries characteristics. $^{21,22}$ Here, we determine the orientational order parameters $\langle P 2\rangle$ and $\langle P 4\rangle$ for both the uniaxial and the biaxial systems. On assuming a specific form of the ODF for the system, we proceed to determine the order parameters, through fits of the analytically derived equations to experimental data. The close fits of the theory to the experiments are used in the methodology for the determinations of the uniaxial and the biaxial order parameters.

\section{EXPERIMENTAL DETAILS}

The molecular structure and the transition temperatures of $\mathrm{MSi}_{3} \mathrm{MR} 11$ obtained by DSC during cooling cycle at the rate $10{ }^{\circ} \mathrm{C} / \mathrm{min}$ are given in Fig. 2. Here the first " $\mathrm{M}$ in $\mathrm{MSi}_{3}$

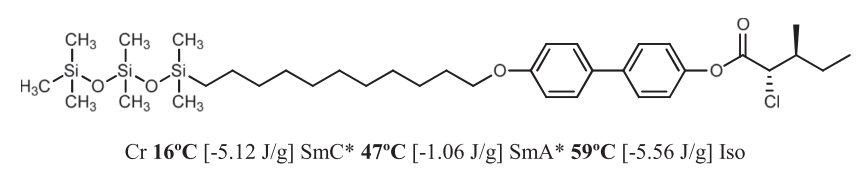

FIG. 2. The chemical structure of the heptamethyltrisiloxane LC compound $\mathrm{MSi}_{3} \mathrm{MR} 11$.

stands for (mono-substituted)," $\mathrm{Si}_{3}$ stands for three siloxane end groups, the second "MR" stands for the initials of the synthetic chemist who first synthesized it. In this compound, the mesogen MR11 with 11 methylene units is attached to trisiloxane backbone. The mesogenic core of MR11 consists of biphenyl 2-chloro-3-methylpropionate.

Homogeneously planar aligned sandwich LC cells were prepared for Raman experiments and homeotropic-aligned cells made for Infrared (IR) spectroscopic investigations. The cell used in these studies was prepared as follows: the compound in its isotropic liquid state is infiltrated into a homogeneously planar-aligned cell formed by the two ITO coated glass windows that were spaced apart by a Mylar spacer of thickness $15 \mu \mathrm{m}$. Prior to it, the ITO coated substrates are spin-coated by polyimide aligning films (Nissan, RN-1266) polymerized using a set procedure, each with a film thickness of approximately $20 \mathrm{~nm}$. Only one of these two film-covered substrates was rubbed in a specific direction to avoid the interface induced electro-clinic effect in the SmA* phase. ${ }^{21} \mathrm{~A}$ homeotropic aligned cell for the IR measurements was prepared from two $\mathrm{ZnSe}$ windows, spacing for the cell used was determined by optical interferometry to be $6.1 \mu \mathrm{m}$. In this case, each window of the cell is coated by chromolane solution to first form a liquid-like thin film of $\sim 30 \mathrm{~nm}$ thick. This film so deposited on the substrate is cured at a temperature of $120^{\circ} \mathrm{C}$. A planar-aligned sample of high quality is obtained by rapid cooling of the cell from the isotropic to $\mathrm{SmA}^{*}$, in the absence of an electric field applied across it. To obtain bookshelf structure in a planar cell, an electric field of $10 \mathrm{~V} / \mu \mathrm{m}$ is applied across the cell. This resulted in an irreversible change from the chevron to bookshelf arrangement of molecules within smectic layers in a planar-aligned cell. A well-aligned uniform area of the cell is selected using the polarized light of Leica microscope, an attachment of the micro-Raman spectrometer ( $\mu R S)$. The Raman spectroscopic experiment was carried out by shining the laser light onto a well-aligned area of the cell.

The texture of the LC in a planar cell under a polarizing microscope is recorded (see Fig. 3) and is observed to be uniformly bright. The color of the texture arises from the cell's birefringence dependent on the LC phase, cell thickness and the temperature. The observed textures confirm the high quality of a planar alignment in the cell. The micro-Raman Spectrometer ( $\mu \mathrm{RS}$ ) (Renishaw RM1000) is equipped with an Ar-ion laser as the source. The laser beam of power, 10 $\mathrm{mW}$, at a wavelength of $488 \mathrm{~nm}$ is used in the backscattering configuration mode (Fig. 4), where the spot size of the laser beam is less than $5 \mu \mathrm{m}$ in diameter. The laser beam is focussed onto an aligned area of the sample selected prior to initiating these measurements, using objective lens of magnification X20 with a long working distance in the microscope. The LC cell is mounted on a LINCAM hot-stage fixed onto a home-made rotational stage of the Leica microscope. The 

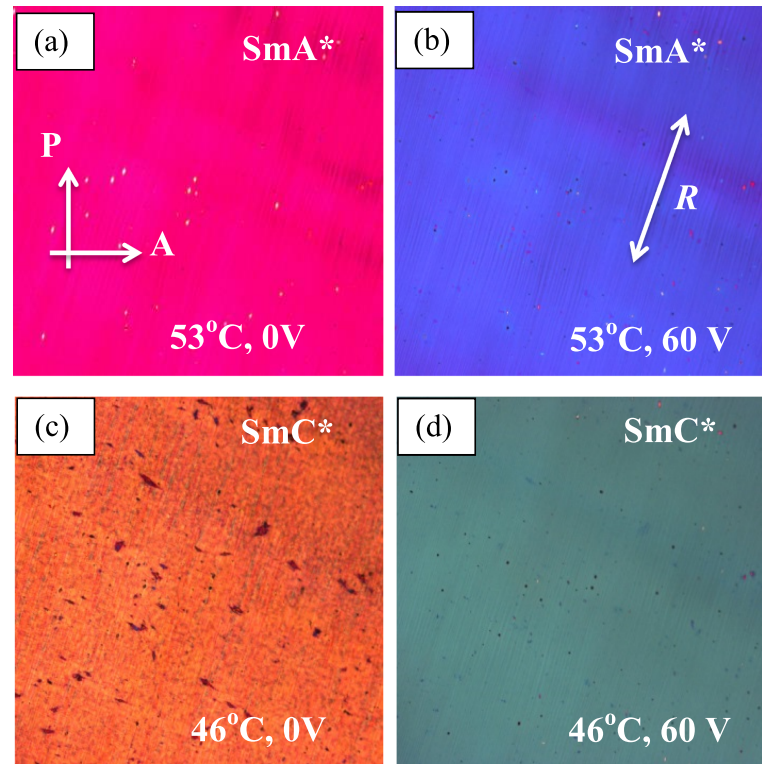

FIG. 3. Polarizing optical microscopic textures of the de Vries SmA* and $\mathrm{SmC}^{*}$ phases. Texture observations are carried out on a $9 \mu \mathrm{m}$ planar aligned cell in different phases filled with the $\mathrm{MSi}_{3} \mathrm{MR} 11$ material. The rubbing direction $\boldsymbol{R}$ in the cell is fixed at an angle of $20^{\circ}$ to the polarizer. Zero field (a) SmA* and (c) $\mathrm{SmC}^{*}$; (b) and (d) square wave AC voltage $\left(60 \mathrm{~V}_{\text {peak to peak }}\right)$ applied across a planar-aligned cell at a frequency of $110 \mathrm{~Hz}$. $\mathbf{P}$ and $\mathbf{A}$ in panel (a) refer to the polarizer and analyser directions, respectively, in the crossed polarized optical microscopy.

rotational stage allowed for the rotation of the optical axis of the sample by an angle of $180^{\circ}$ with respect to the polarisation vector of the incident beam of the laser light. In the scattering mode, the measurements are made for two polarizations of the electric vector of the scattered beam of light: parallel and perpendicular to the polarization vector of the incident laser

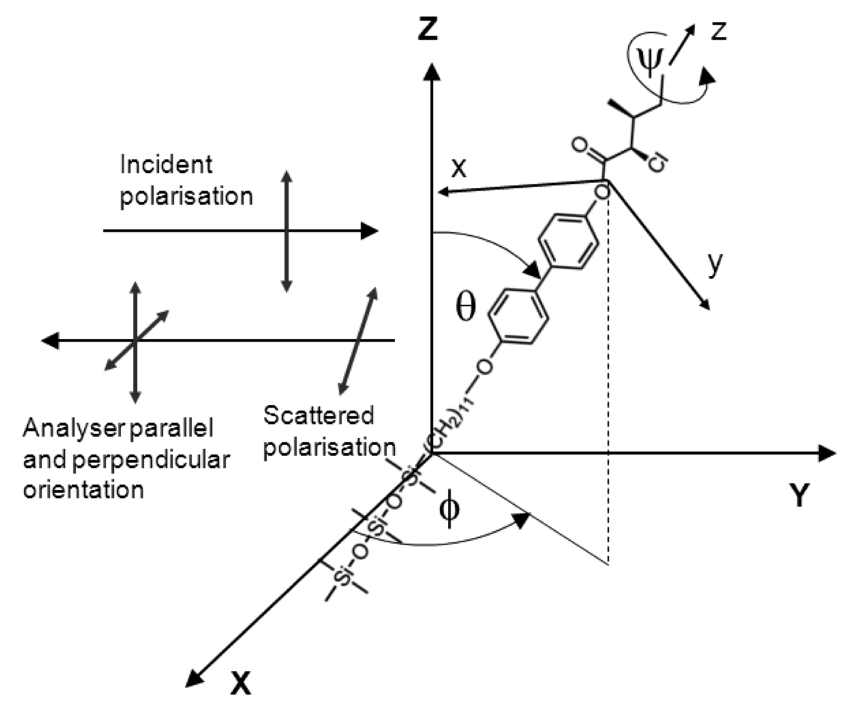

FIG. 4. The schematic orientations of the electric vectors of the incident and scattered beams of light in the polarised Raman spectroscopy are shown. The molecular and the laboratory frames are described by $x, y, z$ and $X, Y$, and $Z$, respectively. In the laboratory frame, $\theta$ is the polar angle and $\phi$ is the azimuthal angle. The angle $\psi$ describes the biased rotation along its long molecular axis. $\omega$ is the angle between the polarisation vector of the incident beam and the $Z$ axis. The laser light is incident along the $Y$ direction, normal to plane of the windows $(X-Z)$ of the cell. Parallel and perpendicular polarizations to the incident light are selected by rotating the analyser by an angle of $90^{\circ}$. light shown in Fig. 4 by rotating the polarizer. The polarized and the depolarized spectra are then measured as a function of the rotation angle $\omega$ of the cell by varying $\omega$ from $0^{\circ}$ to $180^{\circ}$ in steps of $10^{\circ}$ by rotating the table of the spectrometer to which the hot-stage containing the cell is fixed to find the orientational order parameters. $\omega$ is defined as the angle that $Z$ axis of the laboratory frame makes with the polarisation vector of the incident beam of laser light.

The IR absorbance measurements were carried out as a function of temperature from the isotropic state to temperatures deep in the $\mathrm{SmC}^{*}$ phase through steps of $0.5 \mathrm{~K}$ by using a Fourier transform infrared Bio-Rad FTS-6000 spectrometer in its transmission mode. The spectrometer is equipped with a DTGS detector.

\section{RESULTS AND DISCUSSIONS}

\section{A. Polarized Raman spectroscopy of homogeneous planar-aligned cells and the analysis in terms of the uniaxial order parameters}

An experimental unpolarised Raman spectra of a LC homogeneous planar aligned cell filled with $\mathrm{MSi}_{3} \mathrm{MR} 11$ is shown in Fig. 5 together with its simulated spectra in arbitrary units of intensity using Density Functional Theory [DFT B3LYP/6-31G $(d, p)$ ] and a Gaussian 09 software package. ${ }^{23}$ As seen from Fig. 5, the most prominent and the stand-alone Raman line, easily separable from the other spectral lines, is positioned at $1608 \mathrm{~cm}^{-1}$. This line is assigned to the phenyl ring $\mathrm{C}-\mathrm{C}$ stretching vibrations and is used to probe the orientational ordering of molecules. The principal axis with the largest Raman scattering tensor component is almost parallel to the long molecular axis. The depolarization ratio, $R$, is defined as $R=\frac{I_{X}}{I_{Z}}$. In the isotropic state, $R_{\text {iso }}$ calculated using DFT is 0.37 . This is in good agreement with the experimental value of 0.36 .

The measured scattering intensities for the $1608 \mathrm{~cm}^{-1}$ peak versus $\omega$ (the rotation angle) of the cell are shown in Fig. 6 as a linear plot for temperature of $53{ }^{\circ} \mathrm{C}$ and in Fig. 7 as polar plots for temperatures of $36{ }^{\circ} \mathrm{C}, 46{ }^{\circ} \mathrm{C}$, and $56{ }^{\circ} \mathrm{C}$ with the electric field of $+30 \mathrm{~V}$ applied across the cell. The

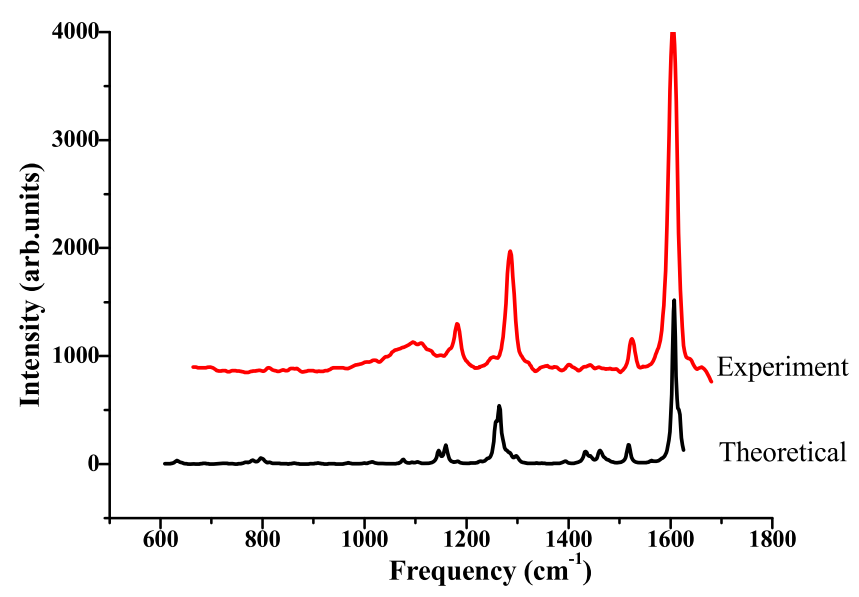

FIG. 5. Raman spectra of the LC compound MSi ${ }_{3}$ MR11, experimental (red curve) and the simulated spectra (black continuous curve, not drawn to scale), are shown. The spectra are simulated using the program given in Ref. 23. 


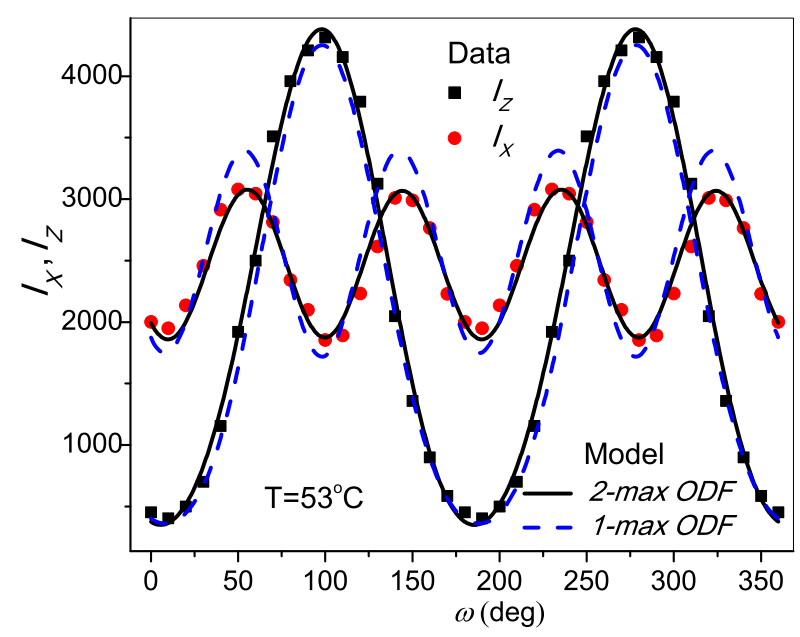

FIG. 6. Linear plots of the polarized Raman scattering intensities (in arbitrary units) for the biphenyl vibration $1608 \mathrm{~cm}^{-1}$ for $I_{Z}$ (squares) and $I_{X}$ (circles) at a temperature of $53{ }^{\circ} \mathrm{C}$ for $+30 \mathrm{~V}$ applied across the planar-aligned cell. $\omega$ is defined as the angle between the $Z$ axis (Fig. 4) and the polarization of the incident light and experimentally is varied in steps of $5^{\circ}$. The experimentally measured relative intensity $I_{X}(\omega)$ is enlarged compared to $I_{Z}(\omega)$ by a factor of 3. Calculated intensities $I_{Z}(\omega)$ and $I_{X}(\omega)$ using Eqs. (1) and (2) are shown by the solid lines in each case when the ODF is fitted to the two maxima. The dashed lines represent fitting of the ODF to a single maximum.

two polarizations of the scattered light with reference to the incident light are illustrated in Fig. 4.

Two approaches have been advanced for describing the orientational order parameters using the Raman technique. Both the conventional (Meier-Saupe mean field model, the sugar-loaf ODF) and the diffuse-cone models for de Vries characteristics, in the absence of electric field, have uniaxial symmetry. Thus the ODF can be expressed in terms of $f(\theta)$ dependent only on the polar angle $\theta$, defined in Fig. 4. The ODF in the laboratory frame is referenced with respect to the $Z$ axis, directed along the layer normal of the bookshelf geometry of the SmA* phase. The function $f(\theta)$ can be expanded into a series of Legendre polynomials $\left\langle P_{n}(\theta)\right\rangle$. The Raman scattering $^{24,25}$ can determine $\left\langle P_{n}(\theta)\right\rangle$ up to $\mathrm{n}=4$, whereas X-ray scattering ${ }^{26,27}$ and the elastic neutron scattering ${ }^{28}$ can find $\left\langle P_{n}(\theta)\right\rangle$ up to $n=6$ provided the alignment produces a single domain. However $\mathrm{NMR}^{29}$ can only determine a set of four scalar order parameters, similar to those found from the IR spectroscopic technique. ${ }^{31}$ In a planar-aligned LC cell, both fields: surface and electric, can induce biaxiality in the $\mathrm{SmA}^{*}$ phase. As a result, the azimuthal angle $\phi$ needs to be included in the ODF, $f(\theta, \phi)$. One then needs to adopt a more general approach of determining the biaxial order parameters, discussed in Sec. III C.

When an electric field is applied across the cell, the azimuths condense and these lie within narrower limits of the angle. On increasing the field further, the fluctuations of the both the polar and the azimuthal angles with respect to the local director are significantly reduced. It is then possible to describe the system by a uniaxial ODF again by tilting the reference direction along the tilt direction. This can then be expressed in terms of $\left\langle P_{n}(\theta)\right\rangle$ in the laboratory frame where the $Z$-axis is directed along the optical axis. When the molecular distribution is cylindrically symmetric as in the $\mathrm{SmA}^{*}$ discussed above, the $Z$ - and $X$-polarized
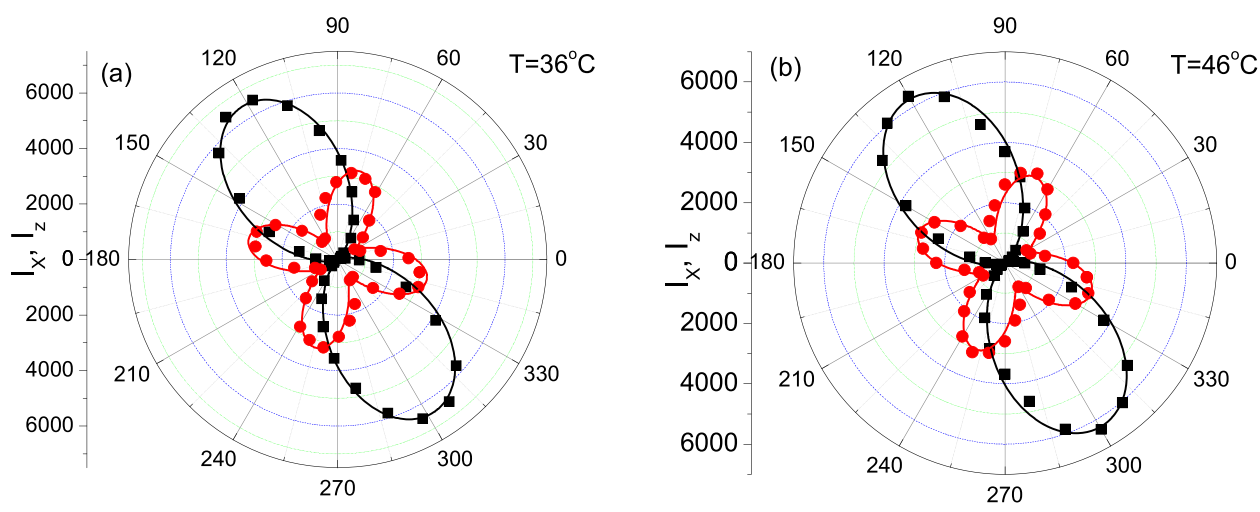

FIG. 7. Polar plots of the polarized Raman scattering vibration at 1608 $\mathrm{cm}^{-1}$ for $I_{Z}$ (filled black squares) and $I_{X}$ (filled red circles) both for an applied voltage of $+30 \mathrm{~V}$ applied across the cell. $\omega$ is defined as the angle the $Z$ axis (Fig. 4) makes with the polarization of the incident light. The cell is rotated to vary $\omega$. The measured value $I_{X, \text { meas }}(\omega)$ in the plot is multiplied by 2 compared to $I_{Z, \text { meas }}(\omega)$. For a temperature of $56^{\circ} \mathrm{C}$, the relative intensities $I_{X, \text { meas }}(\omega)$ and $I_{Z, \text { meas }}(\omega)$ are enlarged by multiplication factors of 1.5 and 3 , respectively. The calculated (fitted) intensities $I_{Z}(\omega)$ and $I_{X}(\omega)$ as per details given in the text are shown by solid lines in each case.

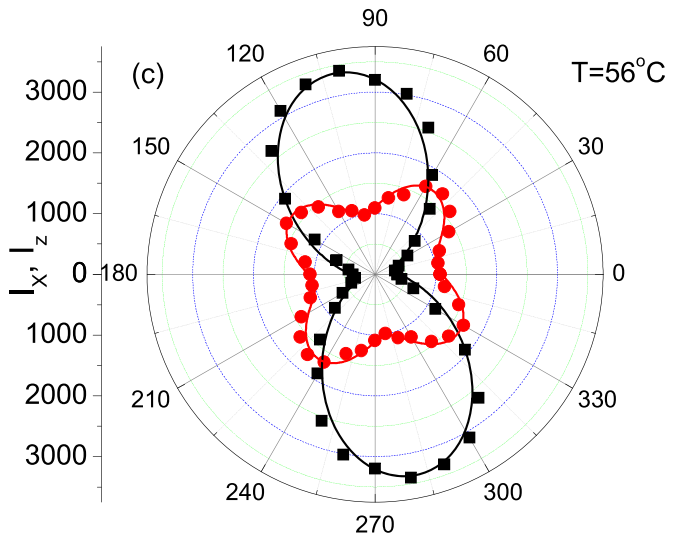


Raman scattered intensities, $I_{Z}(\omega)$ and $I_{X}(\omega)$, can be expressed in terms of Legendre polynomials $\left\langle P_{2}(\cos \theta)\right\rangle$ and $\left\langle P_{4}(\cos \theta)\right\rangle .{ }^{15,16}$ The angle $\omega$ between the polarization vector of the incident laser light and the $Z$ axis (optical axis) (Fig. 4) is varied by rotating the hot-stage, which in turn rotates the $Z$ axis of the sample by an angle $\omega$,

$$
\begin{aligned}
I_{Z}(\omega)= & C_{1}(\omega)+C_{2}(\omega)\left\langle P_{2}(\cos \theta)\right\rangle \\
& +C_{3}(\omega)\left\langle P_{4}(\cos \theta)\right\rangle+C_{4}(\omega) R, \\
I_{X}(\omega)= & C_{5}(\omega)+C_{6}(\omega)\left\langle P_{2}(\cos \theta)\right\rangle \\
& +C_{7}(\omega)\left\langle P_{4}(\cos \theta)\right\rangle-C_{4}(\omega) R .
\end{aligned}
$$

The coefficients $C_{1}$ to $C_{7}$ with periodicity in $\omega, 2 \omega$, etc. are expressed in terms of the transmission coefficients of the incident and scattered light polarized along the $X$ and $Z$ axes, respectively [see, Eqs. (A1) and (A2) in the Appendix]. The depolarization parameter $R$ is given by Eq. (A3) (Appendix). This depends on birefringence, $\Delta n$, sample thickness, $d$, and wavelengths of incident, $\lambda_{i n}$, and scattered beams of the laser light, $\lambda_{s c}$. The experimental data for $I_{Z}(\omega)$ and $I_{X}(\omega)$ profiles are plotted in Fig. 6 as linear and in Fig. 7 as polar plots. The intensities $I_{Z}(\omega)$ and $I_{X}(\omega)$ calculated from Eqs. (1) and (2), with coefficients given in the Appendix, are shown by the solid and dotted lines in Figs. 6 and 7, respectively. $\Delta n$ values used in equations in the Appendix are taken from Ref. 22. In a planar-aligned sample, the azimuthal distributions are strongly affected by several factors: (i) the substrate boundary conditions and (ii) the applied electric field. These separate out the uniform azimuthal distributions into two maxima in the $\mathrm{SmA} *$ phase. An electric field increases the contribution of one profile against the second and finally only one of the two maxima in the azimuthal distribution survives. In that case, the in-layer director $\boldsymbol{n}$ is tilted by $\theta_{\text {Raman }}$ in a single direction (the apparent tilt angle approaches saturation for larger applied fields). A fitting of the experimental data to (i) only one of the two maxima in the ODF and (ii) the two maxima in the ODF are carried out. The latter is applied for cases where some molecules are tilted in one direction and the rest towards the opposite direction. As the electric field across the cell is increased, tilt of molecules in one direction grows at the cost of the second direction. This applies to case (i) and will later be discussed in Fig. 12(c).

In order to determine order parameters $\left\langle P_{2}\right\rangle$ and $\left\langle P_{4}\right\rangle$ from the Raman experiment, the simultaneous fits of $I_{Z}$ and $I_{X}$, in Eqs. (1) and (2) and the constants given in the Appendix, to the experimental scattering data with parameters $\left\langle P_{2}\right\rangle,\left\langle P_{4}\right\rangle$, and $\omega_{M}$, are carried out. $\omega_{M}$ is defined as the angle that the maximum of $I_{Z}$ makes with the optical axis. Quality of the data fitting can be gauzed from the fits shown in Figs. 6 and 7 which is high. In $\mathrm{SmC}^{*}$ phase, the in-layer director $\boldsymbol{n}$ is tilted by $\theta_{\text {Raman }}$ in a single direction, see Fig. 12(c). The tilt direction is anchored onto the substrate-surfaces provided the twist and splay deformations of the director are negligibly small and are thus neglected. In the $\mathrm{SmA}^{*}$ phase, however, a fitting of the ODF with an in-layer director $\boldsymbol{n}$ tilted by $\theta_{\text {Raman }}$ in a single direction does not produce a satisfactory fit of the experimental data, see blue dashed lines in Fig. 6. This demonstrates that $I_{Z}$ and $I_{X}$ profiles cannot be fitted reasonably with the same set of parameters of the ODF, i.e., with the molecular tilt only "in a single direction and sense" with respect to the layer normal.

The $I_{Z}$ and $I_{X}$ profiles in some cases can be considered as superimpositions of the two ODFs with positive $\theta_{\text {Raman }}$ and negative $-\theta_{\text {Raman }}$, with respect to the layer normal [see later the ODF in Fig. 12(b)]. As an example, the fitting at a temperature of $53^{\circ} \mathrm{C}$ is shown in Fig. 6 by solid lines. The chi-square tolerance for the double maxima fit (solid lines in Fig. 6) is better by a factor of four compared to the fit with only a single maximum in the tilt in the ODF (dashed lines in Fig. 6).

Figure 8 shows plots of $\left\langle P_{2}\right\rangle$ and $\left\langle P_{4}\right\rangle$ versus temperature. It is interesting to note that a sharp jump occurs for both $\left\langle P_{2}\right\rangle$ and $\left\langle P_{4}\right\rangle$ at the $\mathrm{SmA}^{*}-\mathrm{SmC}^{*}$ transition temperature. This jump is reminiscent of the first-order nature of the $\mathrm{SmA}^{*}-\mathrm{SmC} *$ transition, confirmed by DSC investigations. ${ }^{22}$ It is to be emphasized here that the order parameters are measured with respect to the optical axis. The optical axis is shifted by the measured apparent tilt angle in the Raman experiment. In such a case, the biaxial order parameters cannot easily be defined as the biaxiality is mainly from the tilt. It must be emphasized that for the IR spectroscopic investigations, the reference direction for the frame is the layer normal.

Figure 9 shows plots of tilt angles: $\theta_{\text {Raman }}$ and $\theta_{\text {optical }}$. $\theta_{\text {optical }}$ is measured using a planar-aligned cell of cell-thickness $3 \mu \mathrm{m}$; across which a large electric field up to $(E=16 \mathrm{~V} / \mu \mathrm{m})$ is applied. We note that on cooling the sample from the isotropic state to the $\mathrm{SmA} *$ phase, $\theta_{\text {Raman }}$, initially stays at almost zero value but it gradually increases as the temperature reaches to within $5^{\circ} \mathrm{C}$ of the $\mathrm{SmA}^{*}-\mathrm{SmC} *$ transition temperature. In this figure, values of $\theta_{\text {Raman }}$ agree closely with $\theta_{\text {optical }}$ in the $\mathrm{SmC}^{*}$ phase though there is a large difference in between the two angles in the $\mathrm{SmA}^{*}$ phase. In the $\mathrm{SmA}^{*}$ phase, we note that there are two values of $\theta_{\text {Raman }}$ depending on the type of fit: either to single or to double maxima in the $I_{Z}$ profile.

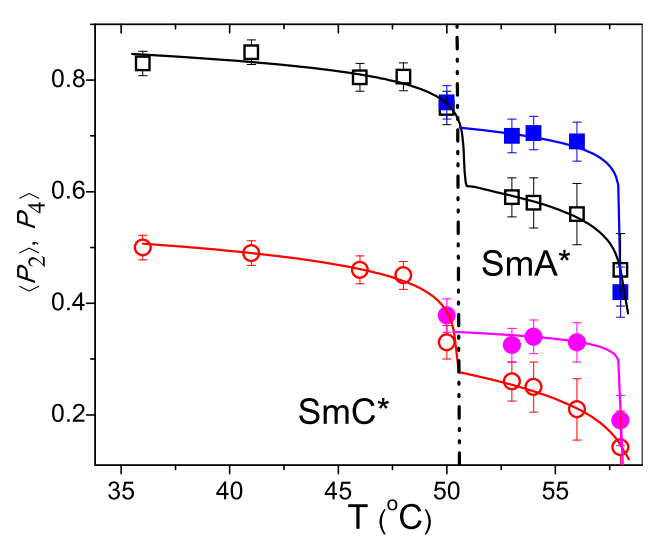

FIG. 8. The Raman orientational order parameters: $\square,\left\langle P_{2}\right\rangle . \bigcirc,\left\langle P_{4}\right\rangle$ obtained by fitting the $I_{Z}$ profile to a single maximum in tilt. Blue filled squares $-\left\langle P_{2}\right\rangle$, pink filled circles, $\left\langle P_{4}\right\rangle$, obtained by fitting the ODF profile to the two maxima in the tilt (calculated for the $1608 \mathrm{~cm}^{-1}$ vibrational band). The solid lines are the fits of the theory [Eqs. (1) and (2)] to the experimental data. At $50{ }^{\circ} \mathrm{C}$ and above, the data are fitted to both single maximum and two maxima, and below this temperature, the data are fitted to a single maximum. The error bars denote the maximum uncertainties in the order parameters. 


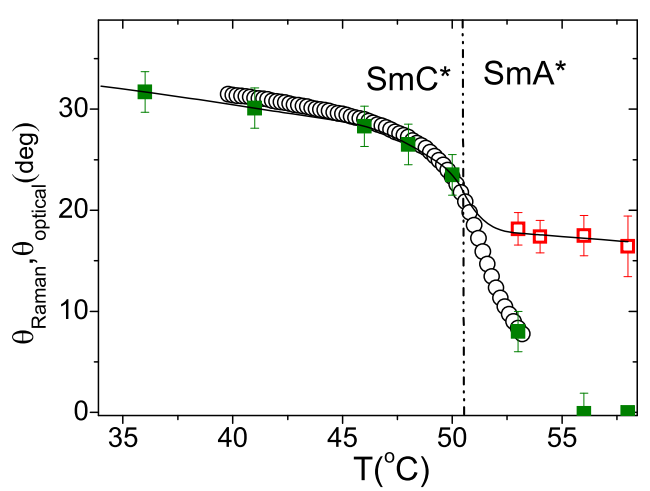

FIG. 9. A comparison of Raman and optical tilt angles shown in the figure. Black open circles are the experimentally measured values of the optical tilt angle as a function of temperature. The filled green squares are $\theta_{\text {Raman }}$ obtained by fitting the $I_{Z}(\omega)$ profile to only one of the two maxima and the red open squares correspond to fitting the profile to two maxima.

\section{B. Infrared spectroscopic studies on a homeotropically aligned cell and the orientational order parameter; comparison of the IR and Raman spectroscopic results}

For a homeotropically aligned cell in the SmA* phase, the IR absorbance is independent of the projection of the director onto the plane of the window; hence the IR polarizer is not required for this experiment. No electric field is applied to the sample in this experiment either. The IR spectra are averaged over 32 scans. The profile of the phenyl ring $\mathrm{C}-\mathrm{C}$ stretching mode at $1608 \mathrm{~cm}^{-1}$ is fitted to a Voigt function for calculating the integrated area, as the "total absorbance of the band." In the SmA* phase, the sample shows axial symmetry about the axis normal to the substrate. The orientational order parameter can be described by the Saupe ordering matrix. ${ }^{30,31}$ The order parameters from the experimentally measured absorbance components of a particular vibrational band ${ }^{31}$ are calculated using the following equation:

$\left(A_{X}+A_{Y}\right) / 2 A_{0}=1+S\left(\frac{3}{2} \sin ^{2} \beta-1\right)+\frac{1}{2} D\left(\sin ^{2} \beta \cos 2 \gamma\right)$,

where

$$
A_{0}=\frac{1}{3}\left(A_{X}+A_{Y}+A_{Z}\right)
$$

$A_{X}, A_{Y}$, and $A_{Z}$ are the absorbance components of the light polarized in the $X, Y$, and $Z$ directions, respectively. $A_{0}$ is the average intensity.

The definition of the various angles involved in Eq. (3) is given in the caption of Fig. 10. $S$ and $D$ are the Saupe order parameters $S_{i j}{ }^{30,31}$ (the orientational and the molecular biaxial parameters, respectively) measured in the laboratory frame of reference where $Z$ axis coincides with the smectic layer normal, $S=S_{z z}^{Z} ; D=S_{x x}^{Z}-S_{y y}^{Z}$. $A_{0}$ is taken from the absorbance measurements in the isotropic phase of the LC under investigation. For the mesogen core, the biphenyl stretching vibrations centred at $\sim 1608 \mathrm{~cm}^{-1}$ or $\sim 1570 \mathrm{~cm}^{-1}$ are chosen. Both of these vibrations lie almost parallel to the phenyl para axis. The ester stretching band $\mathrm{C}=\mathrm{O}$ centred at $\sim 1769 \mathrm{~cm}^{-1}$ can additionally be chosen, its transition dipole moment is almost transverse to the molecular long axis, i.e., $\beta \approx 90^{\circ}$. In some

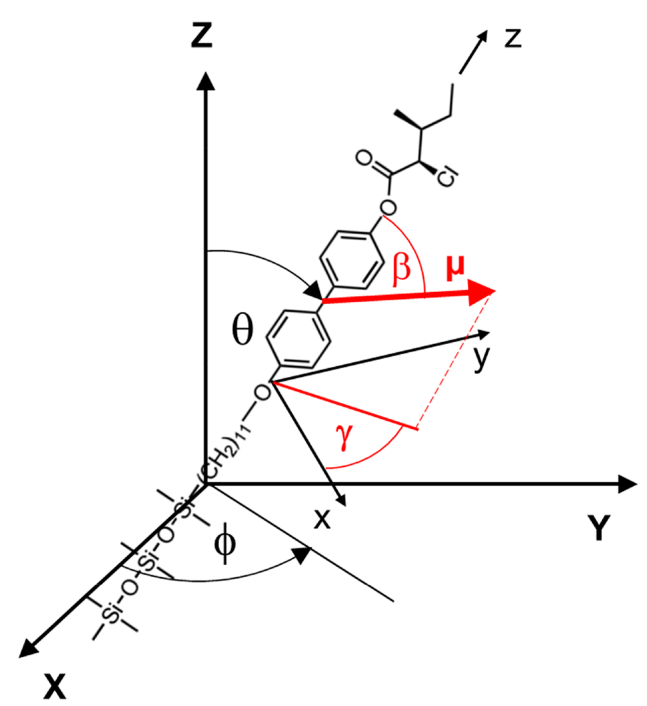

FIG. 10. The laboratory and the molecular frames of reference: $X, Y, Z$ and $x, y$, and $z$. The polar and azimuthal angles: $\beta, \gamma$ are defined in the molecular frame of reference. $\beta$ is the polar angle of the transition dipole moment, i.e., the angle between the molecular axis $z$ and the transition dipole moment $\mu$ of the mode of vibration, $\gamma$ being its azimuthal angle, i.e., the $x$ axis makes with the projection of $\mu$ on the $x$ - $y$ plane (shown as a red line). $\theta$ and $\phi$ are the corresponding polar and azimuthal angles, respectively, in the laboratory frame of reference.

cases, the molecular simulations are used for finding an appropriate value of $\beta$ for a vibrational band of interest. These dominant vibrational bands are determined by the molecular structure. For $1608 \mathrm{~cm}^{-1}, \beta \approx 7^{\circ}$ and for $1769 \mathrm{~cm}^{-1}, \beta \approx 74^{\circ}$, it is convenient to set the $x$ axis in the plane of the ester group $\mathrm{O}-\mathrm{C}=\mathrm{O}$ so that for $1769 \mathrm{~cm}^{-1}, \gamma=0^{\circ}$. If $\beta \approx 0^{\circ}$, we do not need to know the value of $\gamma$ and can thus easily calculate $S$. For finding $D$, the $\mathrm{C}=\mathrm{O}$ stretching vibration band of the ester is used. This has $\beta \approx 74^{\circ} \gamma \approx 0^{\circ}$; again Eq. (3) can be used. The $S$ values of the mesogen part of the molecule are determined and plotted in Fig. 11. The magnitude of $S$ in SmA* is found to be 0.58 . It is rather low compared to an expected value of $\sim 0.8$, Fig. 8. A lower value of $S$ implicitly indicates that the cores of the mesogens are tilted in the $\mathrm{SmA}^{*}$ phase, the distribution of the molecular directors in $\mathrm{SmA}^{*}$ is either uniform or random.

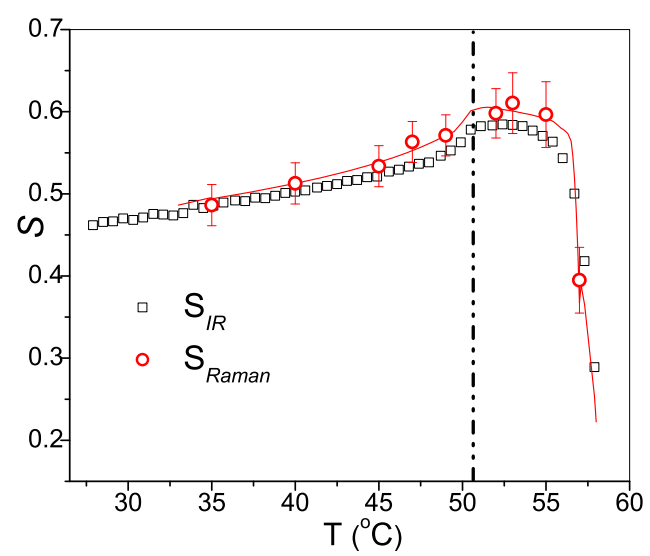

FIG. 11. The Saupe orientational order parameters calculated from the $1608 \mathrm{~cm}^{-1}$ band: $S_{\mathrm{IR}}$, experimental data points $(\square)$ and $S_{\text {Raman }}$, calculated red open circle $(O)$. The error bars in the Raman order parameter specify the maximum uncertainty. Here the frame of reference is Saupe. 
In order to compare the Raman order parameter $\left\langle P_{2}(\cos \theta)\right\rangle$, measured with respect to the optical axis (or the director), with the Saupe order parameter $S_{I R}$ (measured from IR studies in the Saupe frame of reference where the reference axis is the layer normal), one needs to convert the former using the following equation: ${ }^{18,19}$

$$
S_{\text {Raman }}=P_{2}\left(\cos \theta_{\text {Raman }}\right)\left\langle P_{2}(\cos \theta)\right\rangle .
$$

In the Saupe frame, the layer normal is the reference axis, $S_{\text {Raman }}$ values are plotted in Fig. 11, this exhibits an increase in its value in the $\mathrm{SmA}^{*}$ phase with a reduction in temperature as expected. This is followed by a decrease in $S_{\text {Raman }}$ at the $\mathrm{SmA}^{*}-\mathrm{SmC}^{*}$ transition temperature, caused in turn by the tilt of the mesogen away from the layer normal. $S_{\text {Raman }}$ is found to be slightly higher than $S_{\mathrm{IR}}$ ( $S_{I R}$ is the order parameter measured using infrared spectroscopy). For measuring $S_{\text {Raman }}$, an electric field was applied across a planar-aligned cell but for $S_{I R}$, the absorbance data were recorded in the absence of the applied electric field.

\section{A general approach for finding the biaxial orientational order parameters}

Since the phase biaxiality is induced by the surface/electric field in the $\mathrm{SmA}^{*}$ phase, ODF requires both uniaxial and biaxial order parameters to describe the orientational distribution function. The ODF can now be expressed in terms of the generalised Legendre polynomials, $\left\langle P_{l, m, n}\right\rangle$, that include both sets of order parameters, ${ }^{24,25}$

$$
f(\theta, \phi)=\frac{1}{8 \pi^{2}}\left\{\begin{array}{l}
1+\frac{5}{2}\left\langle P_{200}\right\rangle\left(3 \cos ^{2} \theta-1\right)+15\left\langle P_{220}\right\rangle\left(1-\cos ^{2} \theta \cos 2 \phi\right) \\
+\frac{9}{8}\left\langle P_{400}\right\rangle\left(3-30 \cos ^{2} \theta+35 \cos ^{4} \theta\right)+\frac{135}{2}\left\langle P_{420}\right\rangle\left(-1+8 \cos ^{2} \theta-7 \cos ^{4} \theta\right) \cos 2 \phi \\
+\frac{315}{4}\left\langle P_{440}\right\rangle\left(1-2 \cos ^{2} \theta+\cos ^{4} \theta\right) \cos 4 \phi+\cdots
\end{array}\right\},
$$

here the averages are defined as

$$
\begin{aligned}
& \left\langle P_{200}\right\rangle=\frac{1}{2}\left\langle\left(3 \cos ^{2} \theta-1\right)\right\rangle, \\
& \left\langle P_{220}\right\rangle=\frac{1}{4}\left\langle\left(1-\cos ^{2} \theta\right) \cos 2 \phi\right\rangle, \\
& \left\langle P_{400}\right\rangle=\frac{1}{8}\left\langle\left(3-30 \cos ^{2} \theta+35 \cos ^{4} \theta\right)\right\rangle, \\
& \left\langle P_{420}\right\rangle=\frac{1}{24}\left\langle\left(-1+8 \cos ^{2} \theta-7 \cos ^{4} \theta\right) \cos 2 \phi\right\rangle, \\
& \left\langle P_{440}\right\rangle=\frac{1}{16}\left\langle\left(1-2 \cos ^{2} \theta+\cos ^{4} \theta\right) \cos 4 \phi\right\rangle .
\end{aligned}
$$

The expansion of the ODF in Eq. (6) is limited to the polynomials $\left\langle P_{l, m, n}\right\rangle$ up to the order $4 .\left\langle P_{200}\right\rangle$ and $\left\langle P_{400}\right\rangle$ describe the uniaxial order parameters. These are the same as $\left\langle P_{2}\right\rangle$ and $\left\langle P_{4}\right\rangle$, defined earlier. $\left\langle P_{220}\right\rangle,\left\langle P_{420}\right\rangle$, and $\left\langle P_{440}\right\rangle$ are the phase biaxial order parameters defined by Eq. (7). The reference direction is the layer normal in this case. The new definition of the ODF allows for the observed intensity profiles $I_{X}$ and $I_{Z}$ for each of the two analyser orientations to be re-calculated by including the biaxial-order parameters up to the order 4. Importantly, a set of the order parameters even up to the order six may not be enough for reproducing the ODF. In addition, most Raman scattering vibrational bands in the literature are obtained by fitting the profile of the depolarisation ratio $I_{X} / I_{Z}$ and not by fitting both $I_{Z}$ and $I_{X}$ separately as is to be carried out over here. In other words, we fit to the numerator and to the denominator of Eq. (A5), separately. As discussed above, we do not reproduce the ODF using Eq. (6). We instead use the modeled ODF. This is based on Gaussian like dependencies in both the polar and the azimuthal angles, $\theta$ and $\phi$ with a standard deviation in the limits of small $\delta \theta$ and $\delta \phi$, respectively, ${ }^{32}$ as follows:

$$
\begin{aligned}
f(\theta, \phi)= & C \exp \left(-\sin ^{2}\left(\theta-\theta_{0}\right) / \delta \theta^{2}\right) \\
& \times \exp \left(-4 \sin ^{2}\left(\frac{1}{2}\left(\phi-\phi_{0}\right)\right) / \delta \phi^{2}\right)
\end{aligned}
$$

$C$ is a constant in the scaling factor in Eq. (8), defined as

$$
\frac{1}{C}=\int_{0}^{\pi / 2} \int_{-\pi}^{+\pi} f(\theta, \phi) \sin \theta d \theta d \phi .
$$

The proposed ODF of Eq. (8) satisfies the symmetry considerations of the $\mathrm{SmA}^{*}$ phase. It is sufficient to vary the three parameters in the model: $\theta_{0}, \delta \theta$, and $\delta \phi$. The numerical value of $\phi_{0}$ is chosen to be 0 or $\pi$ in SmC* phase, depending on the polarity of the field. The orientational distribution function obeys a $\mathrm{C}_{2}$ symmetry with respect to the azimuthal angle $\phi$ in the $\mathrm{SmA}^{*}$ phase but in the absence of an external electric field. Then we shall have two equivalent maxima at $\theta_{0}$ $=17^{\circ}, \phi_{0}=0$ and $\theta_{0}=17^{\circ}, \phi_{0}=180^{\circ}$. On applying the field, the unfavourable maximum $\left(\phi_{0}=180^{\circ}\right)$ is reduced but the favourable one $\left(\phi_{0}=0\right)$ grows, see Fig. 12, panel (b). When the field is applied, we shall have $C_{2}$ symmetry with respect to the polar angle $\theta$, where $\mathrm{C}_{2}$ axis is perpendicular to the tilt plane in both panels (b) and (c).

The modeled ODF is then used to calculate $\left\langle P_{l, m, n}\right\rangle$. An average $\langle X\rangle$ is defined as the ratio of the integrals of the moments of $f(\theta, \phi)$, 


$$
\langle X\rangle=\frac{\int_{0}^{\pi / 2} \int_{-\pi}^{+\pi} X f(\theta, \phi) \sin \theta d \phi d \theta}{\int_{0}^{\pi / 2} \int_{-\pi}^{+\pi} f(\theta, \phi) \sin \theta d \phi d \theta},
$$

where

$$
X=P_{200}, P_{220}, P_{400}, P_{420}, P_{440} .
$$

The averages $\left\langle P_{200}\right\rangle,\left\langle P_{220}\right\rangle,\left\langle P_{400}\right\rangle,\left\langle P_{420}\right\rangle$, and $\left\langle P_{440}\right\rangle$ are expressed in terms of $\theta_{0}, \delta \theta$, and $\delta \phi$. These are inserted into the equations for the $I_{X}$ and $I_{Z}$ profiles, which now depend on the $\theta_{0}, \delta \theta$, and $\delta \phi$, used into a set of Eq. (A1). These $I_{X}$ and $I_{Z}$ profiles are fitted by these parameters to the experimental data. Finally $\left\langle P_{200}\right\rangle,\left\langle P_{220}\right\rangle,\left\langle P_{400}\right\rangle,\left\langle P_{420}\right\rangle$, and $\left\langle P_{440}\right\rangle$ are calculated for the best fitted values of $\theta_{0}, \delta \theta$, and $\delta \phi$.

At a temperature of $53^{\circ} \mathrm{C}$ in the $\mathrm{SmA} *$ phase, the ODF is given by the fitted values of $\delta \theta=0.53 \operatorname{rad}\left(30^{\circ}\right)$ and $\theta_{0}=0.30$ $\operatorname{rad}\left(17^{\circ}\right)$. Values of the following uniaxial order parameters $\left\langle P_{200}\right\rangle=0.58 \pm 0.02$ and $\left\langle P_{400}\right\rangle=0.15 \pm 0.02$ are obtained in the laboratory co-ordinate system in which the $\mathrm{Z}$ axis is directed along the layer normal and not along the optical axis as in $\mathrm{x}$-ray scattering. These values are related to those given previously $\left(\left\langle P_{2}\right\rangle\right.$ and $\left\langle P_{4}\right\rangle$ in a system where the $Z$-axis lies along the optical axis) as follows: $\left\langle P_{L 00}\right\rangle=P_{L}\left(\cos \theta_{C}\right)\left\langle P_{L}(\cos \theta)\right\rangle$ where $L=2$ or $4, \theta_{c}$ is a cone angle, which here is the same as $\theta_{\text {Raman }}$.

Sanchez-Castillo et al. ${ }^{17}$ formulae reduce to Hayashi et al. ${ }^{15,16,18,19,33}$ when the three biaxial order parameters and the tilt of the frame from the layer normal to the tilt direction are equated to zero. It is emphasized here that Hayashi et al. calculated the order parameters with respect to the optical axis and for this reason they did not need to have the biaxial order parameters as these are de-convoluted by tilting the reference frame, results of Sanchez-Castillo et al. ${ }^{17}$ however are based on the $\mathrm{Z}$ axis lying along the layer normal.

In a planar-aligned sample under the electric field, the ODF in the SmA* phase is not uniaxial but biaxial due to the deformation of the azimuthal distributions given by $\delta \phi$ $=0.82 \mathrm{rad}\left(\right.$ or $\left.47^{\circ}\right)$, Fig. 12(b). The initial uniform azimuthal distribution splits into "two maxima" but as the electric field applied is continually increased, the contribution of one maximum unfavourable to the field is reduced in comparison with the second. This may also significantly increase the average cone angle due to electroclinic effect. A corresponding value of the uniaxial order parameters of the ODF for Fig. 12(c) is given by a set of order parameters: $\left\langle P_{200}\right\rangle=0.49 \pm 0.02$ and $\left\langle P_{400}\right\rangle=-0.05 \pm 0.02$, whereas the biaxial order parameters are given by $\left\langle P_{220}\right\rangle=0.069 \pm 0.005,\left\langle P_{420}\right\rangle=0.033 \pm 0.003$, and $\left\langle P_{440}\right\rangle=0.004 \pm 0.002$. It is interesting to note that $\left\langle P_{400}\right\rangle$ is somewhat slightly negative mainly because $P_{400}\left(\cos \theta_{C}\right)$ becomes negative as $\theta_{C}>30^{\circ}$.

In a homeotropically oriented sample in the SmA* phase, the molecules are tilted from the axis of the cone by an angle $17^{\circ}$ with uniform azimuthal distributions, Fig. 12(a). In the planar sample, however, the azimuthal distributions are strongly affected by the substrate boundary conditions as discussed above, the surface field alone induces biaxiality in the orientational order parameters. An increase in the applied electric field across a planar-aligned cell up to a saturation value of the tilt increases the biaxiality and the average cone angle.

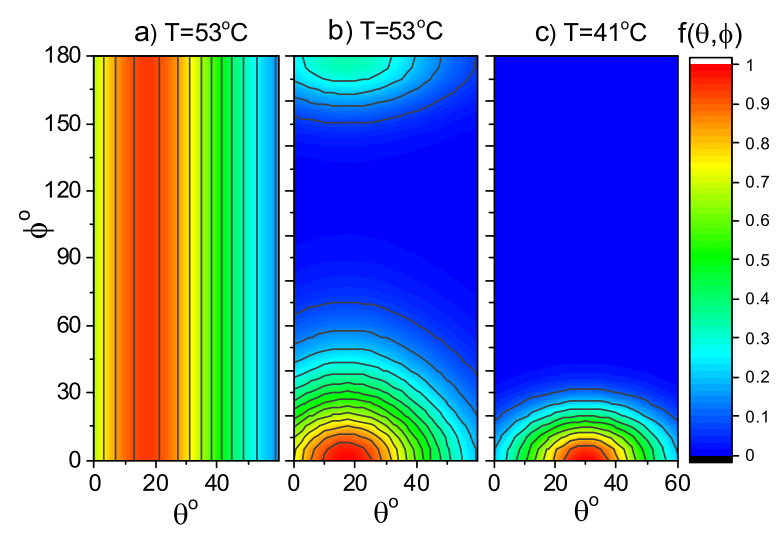

FIG. 12. The orientational distribution function $f(\theta, \phi)$ plotted in both $\mathrm{SmA}^{*}$ and SmC* phases: (a) $f(\theta, \phi)$ is plotted in a homeotropically oriented sample, $\phi$ is uniformly distributed irrespective of the value of $\theta$. This panel is the 2-D version of the 3-D plot shown in Fig. 1(b). The color scale on the right indicates the relative magnitudes of $f(\theta, \phi)$, red corresponds to the highest and blue to the lowest magnitudes (b) the $\operatorname{ODF} f(\theta, \phi)$ in a planar-aligned cell, with a weak electric field applied across the cell, the two maxima of diffuse-cone are seen in panel (b). (c) $f(\theta, \phi)$ plotted for the planar sample with a sufficiently large electric field applied across the cell. In the latter, the field applied is such that the tilt angle is saturated. In this case, the ODF is referenced at a tilt angle of $30^{\circ}$. The color scale for (a) is to be reduced by a factor of 3 , for (b) it is to be reduced by a factor of two, both measured relatively to (c). The maxima in the tilt and azimuthal angles are associated with red subject to the scaling factors for (a)-(c).

\section{CONCLUSIONS}

On the basis of the Raman and IR experiments carried out on a prototypal ferroelectric liquid crystalline material, we find that for a homeotropically oriented sample in the $\mathrm{SmA}^{*}$ phase, the molecules are tilted on the cone by an angle of $17^{\circ}$. In the absence of an external electric field, a uniform azimuthal distribution shown in Fig. 12(a) is found from an analysis of the Raman results, the resulting liquid crystalline system is uniaxial (so long as the azimuths are uniformly distributed on to a cone). In a planar-aligned sample in the $\mathrm{SmA}^{*}$ phase, the molecular distributions are strongly affected by the substrate boundary conditions, where this distribution separates out into two maxima with the result that the system gradually becomes increasingly biaxial. The electric field, when applied, reduces the contribution of one of the two maxima that is unfavourable to it [see Fig. 12(b)] while at the same time significantly increases the average cone angle as well. When the field across the cell is further increased, the tilt finally reaches saturation, the unfavourable maxima out of the two completely vanishes, Fig. 12(c). Based on results of the Raman spectroscopy, we find that the material with de Vries characteristics supports a broad diffuse-cone ODF in the $\mathrm{SmA}^{*}$ phase. This is in line with the simulated volcano-like ODF with a polar tilt angle of $23^{\circ} .{ }^{22}$ An additional important result follows from Fig. 8, where the order parameters $\left\langle P_{2}\right\rangle$ and $\left\langle P_{4}\right\rangle$ jump at the SmA*-SmC* transition. This confirms the first-order nature of the transition already seen by DSC. The order parameters in Sec. III C are calculated on the basis that the $Z$ axis of the laboratory frame is fixed to the layer normal; furthermore calculation of the Saupe order parameters in Sec. III B is also related to such a co-ordinate system. 


\section{ACKNOWLEDGMENTS}

Work of the Dublin and Belfast groups was supported by Award No. 13/US/I2866 from the Science Foundation of Ireland as part of the U.S.-Ireland Research and Development Partnership program jointly administered with the United
States National Science Foundation under Grant No. NSFDMR-1410649. We thank Professor Satyendra Kumar for useful discussions and coordination of the project. The authors (K.M. and A.K.) thank the National Science Centre Poland for Grant No. 2011/03/B/ST3/0336. Yuri Panarin is thanked for discussions.

\section{APPENDIX: ANALYTICAL EXPRESSIONS FOR THE COEFFICIENTS OF THE POLARIZED AND DEPOLARIZED RAMAN SCATTERED INTENSITIES IN TERMS OF ORDER PARAMETERS}

$$
\begin{aligned}
& C_{1}(\omega)=a^{2} T_{X}^{2} T_{X}^{\prime 2}+\frac{4}{45} b^{2} T_{X}^{2} T_{X}^{\prime 2}+\left[-2 a^{2} T_{X}^{2} T_{X}^{\prime 2}+\frac{b^{2}}{45}\left(-8 T_{X}^{2} T_{X}^{\prime 2}+3 T_{Z}^{2} T_{X}^{\prime 2}+3 T_{X}^{2} T_{Z}^{\prime 2}\right)\right] \cos ^{2} \omega \\
& +\left[a^{2}\left(T_{X}^{2} T_{X}^{\prime 2}+T_{Z}^{2} T_{Z}^{\prime 2}\right)+\frac{b^{2}}{45}\left(4 T_{X}^{2} T_{X}^{\prime 2}-3 T_{Z}^{2} T_{X}^{\prime 2}-3 T_{X}^{2} T_{Z}^{\prime 2}+4 T_{Z}^{2} T_{Z}^{\prime 2}\right)\right] \cos ^{4} \omega, \\
& C_{2}(\omega)=-\frac{2}{3} a b T_{X}^{2} T_{X}^{\prime 2}-\frac{4}{63} b^{2} T_{X}^{2} T_{X}^{\prime 2}+\left[\frac{4}{3} a b T_{X}^{2} T_{X}^{\prime 2}+\frac{b^{2}}{63}\left(8 T_{X}^{2} T_{X}^{\prime 2}+3 T_{Z}^{2} T_{X}^{\prime 2}+3 T_{X}^{2} T_{Z}^{\prime 2}\right)\right] \cos ^{2} \omega \\
& +\left[\frac{b^{2}}{63}\left(-4 T_{X}^{2} T_{X}^{\prime 2}-3 T_{Z}^{2} T_{X}^{\prime 2}-3 T_{X}^{2} T_{Z}^{\prime 2}+8 T_{Z}^{2} T_{Z}^{\prime 2}\right)+\frac{a b}{3}\left(-2 T_{X}^{2} T_{X}^{\prime 2}+4 T_{Z}^{2} T_{Z}^{\prime 2}\right)\right] \cos ^{4} \omega \\
& C_{3}(\omega)=\frac{3}{35} b^{2} T_{X}^{2} T_{X}^{\prime 2}+\frac{b^{2}}{35}\left(-6 T_{X}^{2} T_{X}^{\prime 2}-4 T_{Z}^{2} T_{X}^{\prime 2}-4 T_{X}^{2} T_{Z}^{\prime 2}\right) \cos ^{2} \omega+\frac{b^{2}}{35}\left(3 T_{X}^{2} T_{X}^{\prime 2}+4 T_{Z}^{2} T_{X}^{\prime 2}+4 T_{X}^{2} T_{Z}^{\prime 2}+8 T_{Z}^{2} T_{Z}^{\prime 2}\right) \cos ^{4} \omega, \\
& C_{4}(\omega)=T_{X} T_{X}^{\prime} T_{Z} T_{Z}^{\prime} \cos ^{2} \omega-T_{X} T_{X}^{\prime} T_{Z} T_{Z}^{\prime} \cos ^{4} \omega, \\
& C_{5}(\omega)=\frac{b^{2} T_{X}^{2} T_{Z}^{\prime 2}}{15}+\left[a^{2}\left(T_{X}^{2} T_{X}^{\prime 2}+T_{Z}^{2} T_{Z}^{\prime 2}\right)+\frac{b^{2}}{45}\left(4 T_{X}^{2} T_{X}^{\prime 2}-6 T_{X}^{2} T_{Z}^{\prime 2}+4 T_{Z}^{2} T_{Z}^{\prime 2}\right)\right] \cos ^{2} \omega \\
& +\left[a^{2}\left(-T_{X}^{2} T_{X}^{\prime 2}-T_{Z}^{2} T_{Z}^{\prime 2}\right)+\frac{b^{2}}{45}\left(-4 T_{X}^{2} T_{X}^{\prime 2}+3 T_{Z}^{2} T_{X}^{\prime 2}+3 T_{X}^{2} T_{Z}^{\prime 2}-4 T_{Z}^{2} T_{Z}^{\prime 2}\right)\right] \cos ^{4} \omega, \\
& C_{6}(\omega)=\frac{b^{2} T_{X}^{2} T_{Z}^{\prime 2}}{21}+\left[\frac{a b}{3}\left(-2 T_{X}^{2} T_{X}^{\prime 2}+4 T_{Z}^{2} T_{Z}^{\prime 2}\right)+\frac{b^{2}}{63}\left(-4 T_{X}^{2} T_{X}^{\prime 2}-6 T_{X}^{2} T_{Z}^{\prime 2}+8 T_{Z}^{2} T_{Z}^{\prime 2}\right)\right] \cos ^{2} \omega \\
& +\left[\frac{a b}{3}\left(2 T_{X}^{2} T_{X}^{\prime 2}-4 T_{Z}^{2} T_{Z}^{\prime 2}\right)+\frac{b^{2}}{63}\left(4 T_{X}^{2} T_{X}^{\prime 2}+3 T_{Z}^{2} T_{X}^{\prime 2}+3 T_{X}^{2} T_{Z}^{\prime 2}-8 T_{Z}^{2} T_{Z}^{\prime 2}\right)\right] \cos ^{4} \omega \\
& C_{7}(\omega)=-\frac{4}{35} b^{2} T_{X}^{2} T_{Z}^{\prime 2}+\frac{b^{2}}{63}\left(3 T_{X}^{2} T_{X}^{\prime 2}+8 T_{X}^{2} T_{Z}^{\prime 2}+8 T_{Z}^{2} T_{Z}^{\prime 2}\right) \cos ^{2} \omega+\frac{b^{2}}{35}\left(-3 T_{X}^{2} T_{X}^{\prime 2}-4 T_{Z}^{2} T_{X}^{\prime 2}-4 T_{X}^{2} T_{Z}^{\prime 2}-8 T_{Z}^{2} T_{Z}^{\prime 2}\right) \cos ^{4} \omega, \\
& T_{l}=\frac{2 n_{g}}{n_{g}+n_{l}}, \quad T_{l}^{\prime}=\frac{2 n_{l}}{n_{g}+n_{l}} .
\end{aligned}
$$

Here $n_{g}$ is the refractive index of the glass substrate plates and $n_{l}$ is the principal refractive index of the LC with the light polarized along the $l$ axis $(l=X$ or $Z)$. Here we use $n_{g}=1.52, n_{X}=1.5$, and $\Delta n \equiv n_{Z}-n_{X}=\Delta n_{0}\left\langle P_{2}(\cos \theta)\right\rangle$ with $\Delta n_{0}=0.08$, the average $a$ $=\left(2 \alpha_{\perp}+\alpha_{\|}\right) / 3$ and the anisotropy parameter $b=\alpha_{\|}-\alpha_{\perp}$. The Raman scattering tensor components are assumed to be uniaxial. For $1608 \mathrm{~cm}^{-1}$ Raman band: $a=3.91, b=12.6$,

$R=c_{1}\left[2 a^{2}-\frac{4}{45} b^{2}+\left(\frac{2}{3} a b-\frac{8}{63} b^{2}\right)\left\langle P_{2}(\cos \theta)\right\rangle-\frac{8}{35}\left\langle P_{4}(\cos \theta)\right\rangle\right]+c_{2}\left[\frac{2}{15} b^{2}+\frac{2}{21} b^{2}\left\langle P_{2}(\cos \theta)\right\rangle-\frac{8}{35}\left\langle P_{4}(\cos \theta)\right\rangle\right]$.

Here $c_{1}$ and $c_{2}$ depend on sample thickness, $d$, the incident laser light wavelength, $\lambda_{i n}$, and the scattered light wavelength, $\lambda_{s c}$,

$$
c_{1}=\sin \left(K_{1} d\right) / K_{1} d \quad \text { and } \quad c_{2}=\sin \left(K_{2} d\right) / K_{2} d,
$$

with

$$
K_{1}=\frac{2 \pi \Delta n\left(\lambda_{i n}+\lambda_{s c}\right)}{\lambda_{\text {in }} \lambda_{s c}} \quad \text { and } \quad K_{2}=\frac{2 \pi \Delta n\left(\lambda_{i n}-\lambda_{s c}\right)}{\lambda_{\text {in }} \lambda_{s c}} .
$$

Intensity profiles $I_{X}$ and $I_{Z}$ for each of the analyser orientations are 


$$
\frac{I_{X}}{I_{Z}}=\frac{\left(\begin{array}{l}
\left\{3 A h b^{2}\left(m_{1}^{2}-m_{2}^{2}\right) T_{Z}^{2} T_{X}^{\prime 2}\right\} \cos ^{4} \omega+\left\{-6 A b^{2}\left(m_{1}+m_{2}\right) T_{X} T_{X}^{\prime} T_{Z} T_{Z}^{\prime} \sin \left[h\left(m_{1}-m_{2}\right)\right]\right. \\
+h\left(m_{1}^{2}-m_{2}^{2}\right)\left[315\left(T_{X}^{2} T_{X}^{\prime 2}+T_{Z}^{2} T_{Z}^{\prime 2}\right) a^{2}-210\left(E T_{X}^{2} T_{X}^{\prime 2}-2\left\langle P_{200}\right\rangle T_{Z}^{2} T_{Z}^{\prime 2}\right) a b\right. \\
\left.+\left(B T_{X}^{2} T_{X}^{\prime 2}+4 F T_{Z}^{2} T_{Z}^{\prime 2}\right) b^{2}\right]-2\left(315 a^{2}+105 D a b-2 C b^{2}\right) T_{X} T_{X}^{\prime} T_{Z} T_{Z}^{\prime} \\
\left.\left.\sin \left[h\left(m_{1}+m_{2}\right)\right]\right)\right\} \cos ^{2} \omega \sin ^{2} \omega+\left\{3 A h b^{2}\left(m_{1}^{2}-m_{2}^{2}\right) T_{X}^{2} T_{Z}^{\prime 2}\right\} \sin ^{4} \omega
\end{array}\right)}{\left(\begin{array}{l}
\left\{\left(315 a^{2}-210 E a b+B b^{2}\right) h\left(m_{1}^{2}-m_{2}^{2}\right) T_{X}^{2} T_{X}^{\prime 2}\right\}+\left\{h ( m _ { 1 } ^ { 2 } - m _ { 2 } ^ { 2 } ) \left[315\left(T_{X}^{2} T_{X}^{\prime 2}+T_{Z}^{2} T_{Z}^{\prime 2}\right) a^{2}\right.\right. \\
\left.\left.-210\left(E T_{X}^{2} T_{X}^{\prime 2}-2\left\langle P_{200}\right\rangle T_{Z}^{2} T_{Z}^{\prime 2}\right) a b+\left(-3 A T_{Z}^{2} T_{X}^{\prime 2}+4 F T_{Z}^{2} T_{Z}^{\prime 2}+B T_{X}^{2} T_{X}^{\prime 2}-3 A T_{X}^{2} T_{Z}^{\prime 2}\right) b^{2}\right]\right\} \\
\cos ^{4} \omega+\left\{-6 A b^{2}\left(m_{1}+m_{2}\right) T_{X} T_{X}^{\prime} T_{Z} T_{Z}^{\prime} \sin \left[h\left(m_{1}-m_{2}\right)\right] \sin ^{2} \omega\right. \\
+h\left(m_{1}^{2}-m_{2}^{2}\right)\left[630 T_{X}^{2} T_{X}^{\prime 2} a^{2}-420 E T_{X}^{2} T_{X}^{\prime 2} a b+\left(-3 A T_{Z}^{2} T_{X}^{\prime 2}+2 B T_{X}^{2} T_{X}^{\prime 2}-3 A T_{X}^{2} T_{Z}^{\prime 2}\right) b^{2}\right. \\
\left.\left.-2\left(315 a^{2}+105 D a b-2 C b^{2}\right) T_{X} T_{X}^{\prime} T_{Z} T_{Z}^{\prime} \sin \left[h\left(m_{1}+m_{2}\right)\right] \sin ^{2} \omega\right]\right\} \cos ^{2} \omega
\end{array}\right)}
$$

$h$ is the sample thickness, $m_{1}$ and $m_{2}$ are $m_{1}=2 \pi \Delta n / \lambda_{s c}$, $m_{2}=2 \pi \Delta n / \lambda_{i n}$, and the coefficients A-E in formula (A5) are described by generalized Legendre polynominals

$$
\begin{aligned}
A= & 7+5\left\langle P_{200}\right\rangle-30\left\langle P_{220}\right\rangle-12\left\langle P_{400}\right\rangle-180\left\langle P_{420}\right\rangle, \\
B= & 28+20\left\langle P_{200}\right\rangle-120\left\langle P_{220}\right\rangle+27\left\langle P_{400}\right\rangle \\
& +540\left\langle P_{420}\right\rangle+630\left\langle P_{440}\right\rangle, \\
C= & \left.7+10\left\langle P_{200}\right\rangle-60\left\langle P_{220}\right\rangle+18\left\langle P_{400}\right\rangle+270\left\langle P_{420}\right\rangle\right), \\
D= & \left\langle P_{200}\right\rangle-6\left\langle P_{220}\right\rangle, \\
E= & \left\langle P_{200}\right\rangle+6\left\langle P_{220}\right\rangle, F=7+10\left\langle P_{200}\right\rangle+18\left\langle P_{400}\right\rangle,
\end{aligned}
$$

If the biaxial order parameters $\left\langle\mathrm{P}_{220}\right\rangle,\left\langle\mathrm{P}_{420}\right\rangle$, and $\left\langle\mathrm{P}_{440}\right\rangle$ are equated to zero (and the tilt of the frame is also zero), Eqs. (A5) and (A6) then reduce to Eqs. (1) and (2), in which $\mathrm{C}_{1}$ to $\mathrm{C}_{7}$ are given by Eqs. (A1)-(A4).

${ }^{1}$ A. De Vries, Mol. Cryst. Liq. Cryst. 41, 27 (1977).

${ }^{2}$ Yu. P. Panarin, V. Panov, O. E. Kalinovskaya, and J. K. Vij, J. Mater. Chem. 9, 2967 (1999).

${ }^{3}$ C. S. O'Hern, T. C. Lubensky, and J. Toner, Phys. Rev. Lett. 83, 2745 (1999).

${ }^{4}$ A. J. Leadbetter and E. K. Norris, Mol. Phys. 38, 669-686 (1979).

${ }^{5}$ A. de Vries, J. Chem. Phys. 71, 25 (1979).

${ }^{6}$ J. P. F. Lagerwall and F. Giesselmann, ChemPhysChem 7, 20 (2006).

${ }^{7}$ M. V. Gorkunov, F. Giesselmann, J. P. F. Lagerwall, T. J. Sluckin, and M. A. Osipov, Phys. Rev. E 75, 060701R (2007).

${ }^{8}$ K. Saunders, D. Hernandez, S. Pearson, and J. Toner, Phys. Rev. Lett. 98, 197801 (2007).

${ }^{9}$ Y. Shen, L. Wang, R. Shao, T. Gong, C. Zhu, H. Yang, J. E. Maclennan, D. M. Walba, and N. A. Clark, Phys. Rev. E 88, 062504 (2013).

${ }^{10}$ N. A. Clark, T. Bellini, R.-F. Shao, D. Coleman, S. Bardon, D. R. Link, J.

E. Maclennan, X.-H. Chen, M. D. Wand, D. M. Walba, P. Rudquist, and S.

T. Lagerwall, Appl. Phys. Lett. 80, 4097 (2002).

${ }^{11}$ A. Fukuda, in Proceedings of the 15th International Display Research Conference of the SID (Asia Display, 1995), p. 6; S. Inui, N. Iimura, T. Suzuki, H. Iwane, K. Miyachi, Y. Takanishi, and A. Fukuda, J. Mater. Chem. 6, 671 (1996).

${ }^{12}$ S. Jen, N. A. Clark, P. S. Pershan, and E. B. Priestly, J. Chem. Phys. 66, 4635 (1977).

${ }^{13}$ W. J. Jones, D. K. Thomas, D. W. Thomas, and G. Williams, J. Mol. Struct. 708, 145 (2004).

${ }^{14}$ C. D. Southern and H. F. Gleeson, Eur. Phys. J. E 24, 119 (2007).

${ }^{15}$ N. Hayashi and T. Kato, Phys. Rev. E 63, 021706 (2001).
${ }^{16}$ N. Hayashi, T. Kato, T. Aoki, T. Ando, A. Fukuda, and S. S. Seomun, Phys. Rev. E 65, 041714 (2002).

${ }^{17}$ A. Sanchez-Castillo, M. A. Osipov, S. Jagiella, Z. H. Nguyen, M. Kaspar, V. Hamplova, J. Maclennan, and F. Giesselmann, Phys. Rev. E 85, 061703 (2012).

${ }^{18}$ N. Hayashi, T. Kato, A. Fukuda, J. K. Vij, Yu. P. Panarin, J. Naciri, R. Shashidhar, S. Kawada, and S. Kondoh, Phys. Rev. E 71, 041705 (2005).

${ }^{19}$ N. Hayashi, A. Kocot, M. J. Linehan, A. Fukuda, J. K. Vij, G. Heppke, J. Naciri, S. Kawada, and S. Kondoh, Phys. Rev. E 74, 051706 (2006).

${ }^{20}$ Z. Zhang, V. P. Panov, M. Nagaraj, R. J. Mandle, J. W. Goodby, G. R. Luckhurst, J. C. Jones, and H. F. Gleeson, J. Mater. Chem. C 3, 10007 (2015); G. Singh, J. Fu, D. M. Agra-Kooijman, J.-K. Song, M. R. Vengateson, M. Srinivasarao, M. R. Fisch, and S. Kumar, Phys. Rev. E 94, 060701R (2016).

${ }^{21}$ O. E. Panarina, Yu. P. Panarin, F. Antonelli, J. K. Vij, M. Reihmann, and G. Galli, J. Mater. Chem. 16, 842 (2006); N. Yadav, V. P. Panov, V. Swaminathan, S. P. Sreenilayam, J. K. Vij, T. S. Perova, R. Dhar, A. Panov, D. Rodriguez-Lojo, and P. J. Stevenson, Phys. Rev. E 95, 062704 (2017).

${ }^{22}$ S. P. Sreenilayam, D. M. Agra-Kooijman, V. P. Panov, V. Swaminathan, J. K. Vij, Yu. P. Panarin, A. Kocot, A. Panov, D. Rodriquez-Lojo, P. J. Stevenson, M. R. Fisch, and S. Kumar, Phys. Rev. E 95, 032701 (2017).

${ }^{23}$ M. J. Frisch et al., Gaussian 09, Revision E.01, Gaussian, Inc., Wallingford, CT, 2009.

${ }^{24}$ M. S. Park, B. Yoon, J. O. Park, V. Prasad, S. Kumar, and M. Srinivasarao, Phys. Rev. Lett. 105, 027801 (2010).

${ }^{25}$ C. D. Southern, P. D. Brimicombe, S. D. Siemianowski, S. Jaradat, N. Roberts, V. Görtz, J. W. Goodby, and H. F. Gleeson, Eur. Phys. Lett. 82, 56001 (2008).

${ }^{26}$ H. G. Yoon, D. M. Agra-Kooijman, K. Ayub, R. P. Lemieux, and S. Kumar, Phys. Rev. Lett. 106, 087801 (2011).

${ }^{27}$ D. M. Agra-Kooijman, H. G. Yoon, S. Dey, and S. Kumar, Phys. Rev. E 89, 032506 (2014).

${ }^{28}$ R. M. Richardson, J. M. Allman, and G. J. McIntre, Liq. Cryst. 7, 701 (1990).

${ }^{29}$ A. Marchetti, V. Domenici, V. Novotna, M. Lelli, M. Cifelli, A. Lesage, and C. A. Veracini, ChemPhysChem 11, 1641 (2010).

${ }^{30}$ D. A. Dunmur and H. Toriyama, in Handbook of Liquid Crystals, edited by D. Demus, J. Goodby, G. W. Gray, H.-W. Spiess, and V. Vill (Wiley-VCH, Weinheim, 1998), Vol. 1, Chap. VII.3, p. 215.

${ }^{31}$ A. Kocot and J. K. Vij, Liq. Cryst. 37, 653 (2010); A. Kocot, R. Wrzalik, B. Orgasinska, T. Perova, J. K. Vij, and H. T. Nguyen, Phys. Rev. E 59, 551 (1999).

${ }^{32}$ A. Kocot and R. Wrzalik, Relaxation Phenomena (Springer-Verlag, 2003), pp. 445-468.

${ }^{33}$ Y. Yamada, A. Fukuda, J. K. Vij, N. Hayashi, and T. Ando, Liq. Cryst. 42, 864 (2015). 